\title{
CONSTRUCCIÓN DE CURVAS DINÁMICAS DE ÍNDICE DE SITIO PARA Pinus cooperi BLANCO
}

\author{
DEVELOPMENT OF DYNAMIC SITE INDEX CURVES FOR Pinus cooperi BLANCO
}

\author{
Benedicto Vargas-Larreta ${ }^{1 *}$, Juan G. Álvarez-González ${ }^{2}$, J. Javier Corral-Rivas ${ }^{3}$ y \\ Óscar A. Aguirre Calderón ${ }^{4}$
}

\begin{abstract}
${ }^{1}$ Instituto Tecnológico de El Salto. Mesa del Tecnológico s/n. 34942, El Salto, P. N., Durango., México. Tel. 01 (675) 8765509 ext. $105 .{ }^{2}$ Departamento de Ingeniería Agroforestal, Escuela Politécnica Superior. Lugo, España. ${ }^{3}$ Facultad de Ciencias Forestales, Universidad Juárez del Estado de Durango, Río Papaloapan y Blvd. Durango s/n, Col. Valle del Sur. 34120, Durango, México. ${ }^{4}$ Facultad de Ciencias Forestales, Universidad Autónoma de Nuevo León. Carretera Nacional Km. 145. 67700, Linares, Nuevo León.
\end{abstract}

\section{RESUMEN}

En este trabajo se comparan dos procedimientos de ajuste de modelos expresados en diferencias algebraicas generalizadas, para la construcción de curvas dinámicas de índice de sitio basadas en datos procedentes de análisis troncales de árboles dominantes de Pinus cooperi Blanco. La principal ventaja del método de diferencias algebraicas generalizado (GADA) es que la ecuación base puede ser expandida de acuerdo con diversas teorías sobre el crecimiento (p. ej., tasa de crecimiento y asíntota), lo que permite que más de un parámetro de cada modelo dependa de la calidad de estación, que las curvas obtenidas sean más flexibles, y así obtener curvas de índice de sitio que sean a la vez polimórficas y con múltiples asíntotas. El objetivo es obtener curvas que sean invariantes con respecto a la edad de referencia y que estimen directamente la altura dominante y el índice de sitio a cualquier altura y edad. Debido a la estructura longitudinal de los datos empleados, se corrige la dependencia de los errores al considerar la estructura del error como un proceso autorregresivo durante el proceso de ajuste. La ecuación aquí obtenida, derivada del modelo de Chapman-Richards, es muy flexible ya que se puede utilizar para cualquier edad de referencia, sin afectar las predicciones de la altura dominante o del índice de sitio.

Palabras clave: Pinus cooperi, calidad de estación, ecuaciones de diferencias algebraícas generalizadas, modelo autorregresivo continuo.

\section{SUMMARY}

Two fitting procedures for models expressed in generalized algebraic differences are compared to develop dynamic site index curves from stem analysis data corresponding to dominant trees of Pinus cooperi Blanco. The main advantage of the generalized algebraic difference approach (GADA) is that the base equation can be expanded according to various theories of growth (e.g., asymptote and growth rate), thus enabling more than one parameter to be site-specific, allowing the derivation of more flexible dynamic equations, and obtaining polymorphic site-index curves with multiple asymptotes. The objective is to obtain base-age invariant curves that directly estimate height and site index from any height and age. A second-order autoregressive error structure was used in the fitting process to correct the serial correlation of the longitudinal

Recibido: 30 de Marzo del 2009

Aceptado: 18 de Junio del 2010. data used in this study. The resulting equation, derived from the Chapman-Richards model, is very flexible and can be utilized for any reference age without affecting the dominant height or site index predictions.

Index words: Pinus cooperi, site quality, generalized algebraic difference approach, continuous-time autoregressive error structure model.

\section{INTRODUCCIÓN}

La calidad de estación se refiere a la capacidad de un lugar determinado para sustentar el crecimiento de los árboles o de cualquier otra vegetación, y en el caso de bosques también se denomina productividad forestal (Van Laar y Akca, 2007). Las estimaciones precisas de esta productividad son un elemento clave en el manejo forestal, ya que contribuyen a determinar la cosecha anual y el periodo de rotación de las cortas de árboles, además de ayudar a la toma de decisiones acerca de la elección de las especies más adecuadas para una localización concreta. Su cuantificación directa al final del ciclo productivo en términos de volumen de madera es difícil debido a las largas rotaciones de las masas forestales (rodales), y a que sería necesario conocer tanto los volúmenes extraídos en cortas intermedias como los perdidos por causas naturales. Generalmente se usan indicadores intrínsecos al rodal, y la altura dominante es el más adecuado por su baja dependencia de la densidad y de los tratamientos silvícolas habituales, así como por estar estrechamente correlacionada con la producción total en volumen. Para ello se utiliza el índice de sitio, que es la altura media, a una determinada edad de referencia (Clutter et al., 1983).

Los modelos de índice de sitio relacionan la altura y la edad de árboles, y se usan para predecir el desarrollo de 
la altura del rodal y para evaluar la calidad de estación. Las curvas de índice de sitio generadas con estos modelos deben cumplir una serie de propiedades, entre las que destacan las siguientes (Bailey y Clutter, 1974; Cieszewski y Bailey, 2000): polimorfismo, pauta de crecimiento sigmoide con un punto de inflexión, capacidad de alcanzar una asíntota horizontal a edades avanzadas, tener una respuesta lógica (por ejemplo, la altura dominante debe ser cero a la edad cero y la curva debe ser siempre creciente), ser invariantes con respecto al camino de simulación (path invariance) y con respecto a la edad de referencia (base-age invariance). La condición de invarianza con respecto al camino de simulación implica que cuando se parte de la altura dominante $H_{1}$ a la edad $t_{1}$ y se estima con las curvas el valor de la altura dominante $H_{3}$ a la edad $t_{3}$ se debe obtener el mismo valor que si se estima primero la altura dominante $\mathrm{H}_{2}$ a la edad $t_{2}$ y después se emplea este valor para estimar la altura dominante a la edad $t$. La condición de invarianza con respecto a la edad de referencia implica que la forma de las curvas no debe variar, sea cual sea la edad de referencia que se emplee para definir el índice de sitio.

Bailey y Clutter (1974) formalizaron la propiedad de invarianza con respecto a la edad en los modelos de índice de sitio y presentaron una técnica para derivar ecuaciones dinámicas conocida como método de diferencias algebraicas (ADA, algebraic difference approach), que involucra esencialmente la sustitución de un parámetro del modelo base y expresarlo como una función del sitio (en este caso, de una combinación altura dominante-edad). La principal limitación de la metodología $\mathrm{ADA}$ es que la mayoría de los modelos derivados son anamórficos o tienen una asíntota común (Bailey y Clutter, 1974; Cieszewski y Bailey, 2000).

Cieszewski y Bailey (2000) introdujeron una generalización de la metodología ADA, el método de las ecuaciones de diferencias algebraicas generalizado (GADA, generalized algebraic difference approach). La principal ventaja de este método es que la ecuación base puede ser expandida de acuerdo con diversas teorías sobre el crecimiento, como la tasa de crecimiento y asíntota, lo que permite que más de un parámetro de cada modelo dependa de la calidad de estación, y así las familias de curvas obtenidas son más flexibles (Cieszewski y Bailey, 2000; Cieszewski, 2001, 2002, 2003). Con esta generalización se pueden obtener familias que sean a la vez polimórficas y con múltiples asíntotas (Cieszewski, 2002). El objetivo de este trabajo fue construir curvas dinámicas de índice de sitio para Pinus cooperi Blanco en la región de El Salto, Durango, México, mediante dos procedimientos de estimación de parámetros con el método GADA.

\section{MATERIALES Y MÉTODOS}

\section{Área de estudio}

El trabajo se llevó a cabo en la región forestal El Salto, la cual abarca aproximadamente 580000 ha, ubicada en el Estado de Durango, en el noroeste de México, entre $23^{\circ}$ $30^{\prime}$ y $24^{\circ} 15^{\prime}$ de $\mathrm{LN}$ y $105^{\circ} 15^{\prime}$ y $105^{\circ} 45^{\prime}$ de LO. Su altitud varía entre 1400 y $2600 \mathrm{~m}$. La temperatura media anual oscila entre 20 y $22{ }^{\circ} \mathrm{C}$, mientras que la precipitación promedio fluctúa desde 800 hasta $1200 \mathrm{~mm}$ (Corral-Rivas et al., 2004).

\section{Base de datos}

Se utilizaron pares de datos altura dominante-edad, procedentes de análisis troncales de 47 árboles dominantes de Pinus cooperi Blanco. Los árboles fueron derribados y seccionados a la altura del tocón $(0.3 \mathrm{~m})$, a la altura del pecho $(1.3 \mathrm{~m})$ y a intervalos de altura variables (de $1 \mathrm{a}$ $2.5 \mathrm{~m}$ ) a partir de la altura del pecho. La edad de cada sección y alturas verdaderas fueron determinadas en laboratorio a través del algoritmo de Fabbio et al. (1994).

Los valores máximo, mínimo y medio, así como los coeficientes de variación (CV) del diámetro a la altura del pecho (DAP), la altura total y la edad de los árboles muestra, se presentan en el Cuadro 1.

Cuadro 1. Resumen de las variables utilizadas en el ajuste del modelo de índice de sitio para Pinus cooperi Blanco en El Salto, Durango, México.

\begin{tabular}{lccc}
\hline Estadístico & DAP $(\mathrm{cm})$ & Altura $(\mathrm{m})$ & Edad (años) \\
\hline Mínimo & 5.8 & 10.3 & 34 \\
Máximo & 30.0 & 30.0 & 161 \\
Media & 19.1 & 20.5 & 94.6 \\
CV $(\%)$ & 23.9 & 22.0 & 34.5 \\
\hline
\end{tabular}

DAP = diámetro a la altura del pecho; $\mathrm{CV}=$ coef. de variación.

\section{Descripción de la metodología GADA}

El primer paso en el desarrollo de un modelo en forma GADA es seleccionar una ecuación base e identificar el número de parámetros de esa ecuación que serán dependientes de la productividad del sitio. A continuación estos parámetros deben expresarse como funciones de la estación definida por una variable $X$ (variable no observable e independiente que describe la productividad del sitio como resultado de la suma de factores como regímenes de manejo, condiciones de suelo, y factores ecológicos y climáticos) y nuevos parámetros. De esta forma, la ecuación base bidimensional inicialmente 
seleccionada $[(H=f(t))]$ es expandida en una ecuación explícita tridimensional de índice de sitio $[(H=f(t, X))]$. Dado que $X$ no puede ser medida en la realidad o definida funcionalmente, el paso final del procedimiento GADA implica despejar el valor de $X$ a partir de condiciones iniciales de la estación, es decir, de valores de partida de edad y altura dominante, to y $H_{0}\left(H=f\left(t, t o, H_{0}\right)\right.$, de forma que el modelo pueda ser definido implícitamente y aplicable en la práctica (Cieszewski y Bailey, 2000; Cieszewski, 2002).

En este trabajo se utilizó el modelo de ChapmanRichards (Richards, 1959), el cual es muy flexible y ha sido ampliamente utilizado en la construcción de curvas de índice de sitio y que relaciona la altura dominante con la edad. Este modelo tiene asíntota horizontal, sigue un patrón de crecimiento sigmoide y tiene un punto de inflexión. Su forma general puede ser representada como:

$$
H=a_{1}\left[1-e^{a_{2} t}\right]^{a_{3}}
$$

donde $H$ es la altura dominante (m), $t$ es la edad (años) y $a_{1}, a_{2}$ y $a_{3}$ son los parámetros del modelo. Con la notación general de las formulaciones GADA, $a_{1}, a_{2} \ldots a_{n}$ se refieren a los parámetros del modelo base, esto es, parámetros locales que cambian de un sitio a otro, mientras que $b_{1}, b_{2} \ldots b_{m}$ son los parámetros globales que permanecen constantes para todos los sitios. Todas las formulaciones de los modelos GADA tienen la forma general

$$
Y=f\left(t, t_{0}, Y_{0}, b_{1}, b_{2}, \ldots b_{m}\right)
$$

donde $Y$ es el valor de la función a la edad $t$, y $Y_{0}$ es la variable de referencia definida como el valor de la función a la edad $t$.

Para derivar un modelo polimórfico con múltiples asíntotas a partir del modelo de Chapman-Richards antes definido (Ec. 1), más de un parámetro debe ser una función de la productividad del sitio. No es posible obtener alguna expresión matemática de un modelo GADA en el que el parámetro $a_{2}$ varíe con la calidad de estación, puesto que cuando $a_{1}$ o $a_{3}$ es una función de $X$ y el parámetro $a_{2}$ también es una función de $X$, el modelo no puede ser resuelto para $X$ (Cieszewski, 2004). Entonces, en el modelo derivado de la Ec. 1 con formulación GADA, tanto la asíntota $a_{l}$ como el parámetro de forma $a_{3}$ se pueden considerar dependientes de la calidad de estación $(X)$ y dicha relación se puede expresar de la siguiente forma:

$$
a_{1}=X, \quad a_{3}=b_{2}+\frac{b_{3}}{X} \quad \text { y } \quad a_{2}=b_{1}
$$

[Ec. 2]
De este modo, para dos pares de valores altura dominante-edad, la Ec. 1 se reescribe como:

$$
\begin{aligned}
& H_{0}, t_{0} \Rightarrow H_{0}=e^{X} \cdot\left(1-e^{-b_{1} t_{0}}\right)^{b_{2}+b_{3} / X} \\
& H_{1}, t_{1} \Rightarrow H_{1}=e^{X} \cdot\left(1-e^{-b_{1} t_{1}}\right)^{b_{2}+b_{3} / X}
\end{aligned}
$$

donde $H_{0}$ es la altura dominante a la edad inicial to y $H_{l}$ es la altura dominante a la edad $t_{l}$. Al tomar logaritmos (ln) a ambos lados de la primera de las igualdades anteriores, se obtiene la siguiente igualdad:

$$
\ln \left(H_{0}\right)=X+\left(b_{2}+b_{3} / X\right) \cdot \ln \left(1-e^{-b_{1} \cdot t_{0}}\right)
$$

Para esta ecuación, la solución de $X$ involucra encontrar las raíces de una ecuación cuadrática y seleccionar la expresión de la raíz más apropiada para después sustituirla en la segunda expresión del par de ecuaciones [Ecs. 3]. Al reordenar los términos de la ecuación anterior se obtiene un polinomio de segundo grado en función de $X$ cuyo valor se puede despejar. La solución para $X$ en la Ec. 4 con valores de condición iniciales to y $H$, es:

$$
X_{0}=\frac{1}{2}\left\{\ln \left(H_{0}\right)-b_{2} L_{0} \pm \sqrt{\left[b_{2} L_{0}-\ln \left(H_{0}\right)\right]^{2}-4 b_{3} L_{0}}\right\}
$$

donde $\quad L_{0}=\ln \left[1-e^{\left(-b_{1} t_{0}\right)}\right]$

Al seleccionar la solución adecuada de $X_{0}$ (en este caso la que involucra el valor positivo de la raíz cuadrada) y sustituirla en la segunda igualdad de las Ecs. 3, se obtiene la siguiente ecuación dinámica en diferencias algebraicas generalizadas que proporciona curvas polimórficas con múltiples asíntotas:

$$
H_{1}=H_{0} \cdot\left[\frac{1-e^{-b_{1} t_{1}}}{1-e^{-b_{1} t_{0}}}\right]^{b_{2}+b_{3} / X_{0}}
$$

donde $X_{0}$ viene dado por la Ec. 5. El ajuste de esta ecuación a datos reales altura dominante-edad permite estimar los valores de los parámetros globales $b_{1}, b_{2}$ y $b_{3}$.

Todas las familias de curvas obtenidas con el método de ecuaciones de diferencias algebraicas o su generalización, son invariantes con respecto a la edad de referencia e invariantes con respecto al camino de simulación. Para más detalles sobre la formulación de ecuaciones en diferencias algebraicas generalizadas se pueden revisar los trabajos de Cieszewski (2000, 2001, 2002, 2003 y 2004). 


\section{Análisis estadístico}

El uso de datos originales procedentes de análisis troncales en análisis de regresión, normalmente lleva a rechazar la hipótesis de partida de que los errores son independientes, debido a que introduce automáticamente una falta de independencia entre las observaciones (Parresol y Vissage, 1998). Este problema no afecta a las estimaciones de los parámetros, aunque sí a la estimación de sus errores estándar y, por tanto, a cualquier inferencia que quiera hacerse con base en esos errores estándar (Goelz y Burk, 1992).

El potencial problema de autocorrelación de los datos se solucionó con el método de mínimos cuadrados generalizados no lineales (Monserud, 1984; Goelz y Burk, 1992; Huang, 1997), y con el término del error expandido mediante un modelo autorregresivo continuo de segundo orden $(\mathrm{CAR}(2))$. Esta estructura del error permite aplicar los modelos a datos irregularmente espaciados, es decir, datos de parcelas en las que el intervalo de medición no es siempre el mismo, o datos de análisis troncales en los que las edades a las que se conoce la altura dominante no están equidistantes (Gregoire et al., 1995; Zimmerman y Núñez-Antón, 2001). En un modelo autorregresivo continuo de orden 2, la expresión de la estructura del error es la siguiente:

$$
H_{i j}=f\left(H_{j}, t_{i}, t_{j}, \beta\right)+e_{i j} \operatorname{con} e_{i j}=d_{1} \rho_{1}^{t_{i j}-t_{i j-1}} e_{i j-1}+d_{2} \rho_{2}^{t_{i j}-t_{j j}-2} e_{i j-2}+\varepsilon_{i j}
$$

donde $H_{i j}$ representa la predicción de la altura $i$ mediante $H_{j}$ (altura $j$ ), $t_{i}$ (edad $\left.i\right)$, y $t_{j}$ (edad $j \neq i$ ) como variables predictoras; $\beta$ es el vector de parámetros a estimar; $e_{i j}$ es el correspondiente término del error; $d_{l}=1$ para $j>1 \mathrm{y}$ cero cuando $j=1, d_{2}=1$ para $j>2$ y cero cuando $j \leq 2 ; \quad \rho_{1}$ y $\rho_{2}$ son los parámetros autorregresivos de primer orden y segundo orden respectivamente, cuyos valores deben estimarse; $t_{i j-} t_{i j-1}$ es la distancia temporal que separa la observación $j_{t h}$ de la observación $j_{t h-1}$ en cada análisis troncal; $t_{i j}>t_{i j-1}$, y $t_{i j-} t_{i j-2}$ es la distancia temporal que separa la observación $j_{t h}$ de la observación $j_{t h-2}$ en cada análisis troncal $t_{i j}>t_{i j-2}$. Los términos de error para estructuras autorregresivas de mayor orden se expanden de modo similar. En todos los casos $\varepsilon_{i j}$ son ahora errores independientes.

El ajuste simultáneo de la estructura de la media (dada por la ecuación de crecimiento) y de la estructura del error (dada por el modelo autorregresivo) se hizo con el procedimiento MODEL del paquete estadístico
SAS/ETS ${ }^{\circledR}$ (SAS Institute, 2004), que permite una actualización dinámica de los residuos.

El análisis de la capacidad de ajuste de los modelos se basó en comparaciones numéricas y gráficas. Así, a partir de los residuos obtenidos en la fase de ajuste se calcularon el sesgo $(\bar{E})$, la raíz del error medio cuadrático $(R E M C)$ y el coeficiente de determinación ajustado $\left(R_{a d j}^{2}\right)$. Las comparaciones gráficas son necesarias para comprobar que las curvas se ajusten a los datos en todo su rango; además, diferentes modelos pueden presentar los mismos estadísticos de comparación, pero con una respuesta distinta. Entre los métodos gráficos disponibles se emplearon los siguientes: (1) Superposición de las curvas ajustadas sobre las trayectorias de las alturas observadas a lo largo del tiempo, (2) Representación de los residuos frente a los valores predichos por el modelo, y (3) Análisis de la evolución del sesgo y del error medio cuadrático para las distintas clases de edad.

\section{Procedimientos de ajuste para la estimación de parámetros}

En este trabajo se utilizaron dos métodos invariantes respecto a la edad de referencia (base-age invariance), que estiman los efectos específicos del sitio y suponen que los datos siempre contienen errores de medición y errores aleatorios, los cuales deben ser modelados (Cieszewski, 2003). Esos dos métodos de estimación de parámetros son el método de variables artificiales o 'dummy' (dummy approach) propuesto por Cieszewski et al. (2000) y el método iterativo (nested iterative procedure) descrito por Tait et al. (1988). Además, para conocer la ganancia estadística que significa modelar la estructura del error a través de un modelo autorregresivo, los resultados se compararon con los obtenidos con un modelo que no considera la corrección de la autocorrelación de los errores.

En el método de variables dummy las condiciones iniciales $\left(t_{0}, \mathrm{H}_{0}\right)$ son idénticas para todas las mediciones que pertenecen al mismo árbol. La edad inicial puede ser seleccionada arbitrariamente, incluso para cada árbol, aunque con la condición de que sea distinta de cero. A continuación, la altura correspondiente a la edad inicial se estima simultáneamente para cada árbol (que genera un parámetro único para cada individuo) junto con todos los parámetros del modelo global. En este método se requieren un mínimo de dos mediciones por sitio o árbol, y el número de sitios o árboles debe ser mayor que el número de parámetros globales en el modelo.

En este sentido, en la Ec. 6 la variable inicial $H_{o}$ debe ser sustituida por una suma de términos que contienen un 
parámetro dependiente de la calidad de estación (una altura inicial $H_{0 i}$ para el árbol $i$ ), y una variable dummy, en este caso para cada árbol:

$$
H_{1}=\left(H_{01} I_{1}+H_{02} I_{2}+H_{03} I_{3}+\cdots+H_{0 n} I_{n}\right) \cdot\left[\frac{1-e^{-b_{1} \cdot t_{1}}}{1-e^{-b_{1} \cdot t_{0}}}\right]^{b_{2}+b_{3} / X}
$$

donde $I_{i}$ es una variable dummy igual a 1 para el árbol $i$ y 0 en caso contrario. La suma de términos de las edades iniciales multiplicadas por las variables dummy da lugar, durante el proceso de ajuste, a un único parámetro para cada árbol, es decir, una altura estimada a la edad inicial especificada (Cieszewski et al., 2000).

El segundo método de ajuste utiliza un procedimiento iterativo para estimar los parámetros dependientes del sitio y los globales (Tait et al., 1988). El procedimiento inicia con la estimación de los parámetros globales $\left(b_{1}, b_{2}\right.$ y $b_{3}$ en la Ec. 6), al considerar constante el parámetro local $\left(H_{0}\right)$, que varía para cada árbol y al que inicialmente se le asigna como valor la altura media observada a una edad base dada. En el segundo paso, los valores estimados de los parámetros globales se consideran como constantes y el parámetro local $\left(H_{0}\right)$ se estima para cada árbol y se ajusta nuevamente el modelo al emplear, como valor inicial para los parámetros locales, la altura observada a la edad media del árbol. Posteriormente, el valor estimado del parámetro local para cada árbol se considera nuevamente una constante y se ajusta el modelo para estimar de nuevo los parámetros globales. El procedimiento se repite hasta que las estimaciones sucesivas de los parámetros globales se estabilizan. En este caso se utilizó como criterio el que la reducción del error medio cuadrático del modelo fuera menor de 0.0001 .

\section{RESULTADOS Y DISCUSIÓN}

Para detectar problemas de autocorrelación entre los residuos de un mismo árbol se efectuó el ajuste de la Ec. 6 sin tener en cuenta los parámetros de la estructura de autocorrelación del error $\left(\rho_{i}\right)$, y se calculó el valor del estadístico de Durbin-Watson. El valor obtenido para este estadístico (0.41) evidenció la presencia de autocorrelación de los errores. Posteriormente se hizo un ajuste simultáneo de la estructura de la media y del error con un modelo autorregresivo de orden 2 (CAR(2)), y se obtuvo un valor del estadístico de Durbin-Watson de 1.94 (muy próximo a 2), con lo que se demuestra la corrección de la dependencia de residuos.
La Figura 1 (primera fila) muestra la tendencia de los residuales al ajustar la Ec. 6 sin tener en cuenta la autocorrelación de los errores. Después de la corrección de la autocorrelación con el modelo autorregresivo de segundo orden, la tendencia en los residuales desaparece (Figura 1 tercera fila).

Al igual que Cieszewski (2001), se obtuvieron resultados similares con ambos procedimientos de ajuste (dummy e iterativo). Los parámetros estimados para la Ec. 6 en cada procedimiento, así como los estadísticos de ajuste, se muestran en el Cuadro 2. Todos los parámetros fueron significativos a un nivel de $1 \%$, incluyendo los parámetros dependientes del sitio para cada árbol.

El modelo que considera la corrección de la autocorrelación de los errores explica $99.1 \%$ de la varianza total y provee un patrón aleatorio de los residuos alrededor de la línea del cero con varianza homogénea y sin detectarse ninguna tendencia significativa, tanto en la predicción de alturas como de índices de sitio (Figura 2).

También se analizó la tendencia del sesgo y la raíz del error medio cuadrático en la estimación de las alturas para diferentes clases de edad (Figura 3).

La estimación de alturas con la ecuación que no considera la corrección de la autocorrelación presenta un sesgo mucho mayor casi en todas las clases de edad, sobre todo en edades jóvenes, mientras que la ecuación que considera la estructura del error mostró siempre una distribución del sesgo alrededor de la línea del cero, lo cual coincide con lo reportado por Diéguez-Aranda et al. (2006) y por Corral-Rivas et al. (2004). La raíz del error medio cuadrático $(R E M C)$ también es bastante más grande para todas las clases de edad cuando se estiman las alturas sin considerar la corrección de la autocorrelación.

En la Figura 4 se muestran las curvas obtenidas para índices de sitio de 7, 12, 17 y $22 \mathrm{~m}$ a la edad de 50 años, superpuestas sobre los gráficos de perfil de los árboles empleados en el ajuste. Este gráfico permite verificar que las curvas ajustadas siguen la tendencia de los datos en todo el rango de edades, puesto que los modelos con los mismos estadísticos de comparación en ocasiones presentan formas de las curvas bastante distintas (unas subestiman en las primeras edades y sobreestiman a edades avanzadas y viceversa). En la Figura 4 se observa que las curvas describen trayectorias de crecimiento en altura dominante de manera plausible para todas las edades. 

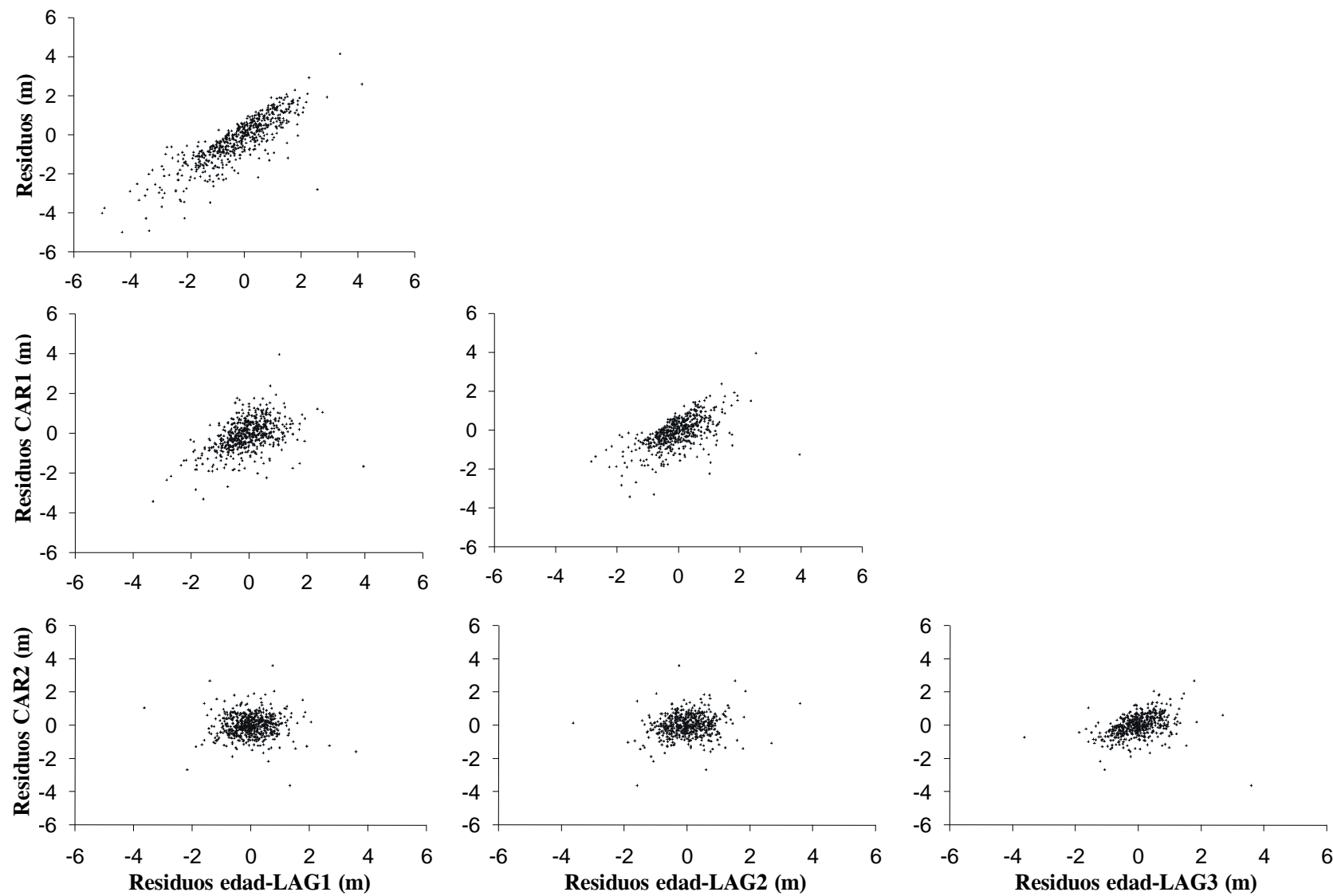

Figura 1. Residuos frente a: residuos de la observación anterior (columna de la izquierda), residuos de la observación realizada dos mediciones antes (columna central), y residuos de la observación realizada tres mediciones antes (columna de la derecha) para la Ec. 6 $\left(H_{1}=H_{0} \cdot\left[\frac{1-e^{-b_{1} \cdot t_{1}}}{1-e^{-b_{1} t_{0}}}\right]^{b_{2}+b_{3} / X_{0}}\right)$ ajustada sin considerar los parámetros autorregresivos (primera fila), mediante un modelo autorregresivo continuo de orden 1 (segunda fila) y con un modelo autorregresivo de orden 2 (tercera fila).

Cuadro 2. Parámetros estimados y estadísticos de ajuste para la Ec. $6^{\dagger}$ en cada procedimiento de estimación de parámetros, comparados con los obtenidos con el modelo que no considera la corrección de la autocorrelación de los errores.

\begin{tabular}{lcccccc}
\hline Ajuste & Parámetro & Valor estimado & $\begin{array}{c}\text { Error est. } \\
\text { aprox. }\end{array}$ & Valor- $t$ & $\begin{array}{l}\text { Valor- } p \\
\text { aprox. }\end{array}$ & REMC \\
\hline Sin corrección & $b_{1}$ & 0.0188 & 0.0005 & 36.77 & $<.0001$ & 1.1156 \\
& $b_{2}$ & -1.7028 & 0.1094 & -15.57 & $<.0001$ & 0.9764 \\
Método & $b_{3}$ & 10.6584 & 0.3688 & 28.90 & $<.0001$ & 0.6788 \\
dummy & $b_{1}$ & 0.0191 & 0.0013 & 15.22 & $<.0001$ & 0.9919 \\
& $b_{2}$ & -1.7919 & 0.7041 & -2.54 & $<.0001$ & \\
& $b_{3}$ & 11.1035 & 2.4411 & 4.55 & $<.0001$ & \\
Método & $\rho_{1}$ & 1.0178 & 0.0026 & 393.50 & $<.0001$ & \\
iterativo & $\rho_{2}$ & 0.9801 & 0.0018 & 554.06 & $<.0001$ & 0.6659 \\
& $b_{1}$ & 0.0174 & 0.0008 & 22.95 & $<.0001$ & \\
& $b_{2}$ & -1.9050 & 0.1796 & -10.61 & $<.0001$ & \\
& $b_{3}$ & 11.2228 & 0.6083 & 18.45 & $<.0001$ & \\
& $\rho_{1}$ & 1.0158 & 0.0024 & 425.23 & $<.0001$ & \\
& $\rho_{2}$ & 0.9794 & 0.0016 & 606.53 & $<.0001$ & \\
\hline
\end{tabular}

$H_{1}=H_{0} \cdot\left[\frac{1-e^{-b_{1} t_{1}}}{1-e^{-b_{1} t_{0}}}\right]^{b_{2}+b_{3} / X_{0}}$

; REMC = raíz del error medio cuadrático; $\mathrm{R}^{2}=$ coeficiente de determinación 

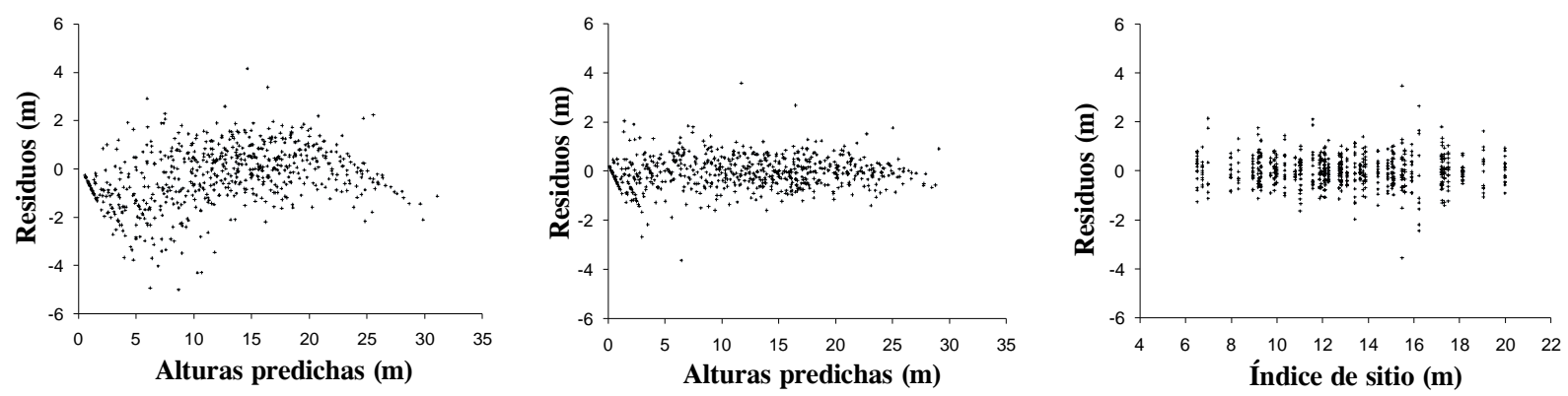

Figura 2. Residuos frente a: alturas predichas como una ilustración de potencial heterocedasticidad (izquierda), alturas predichas reales (centro) e índices de sitio a la edad de referencia de 50 años (derecha) al utilizar la Ec. 6 ajustada con el modelo autorregresivo de la estructura del error de segundo orden (CAR(2)).
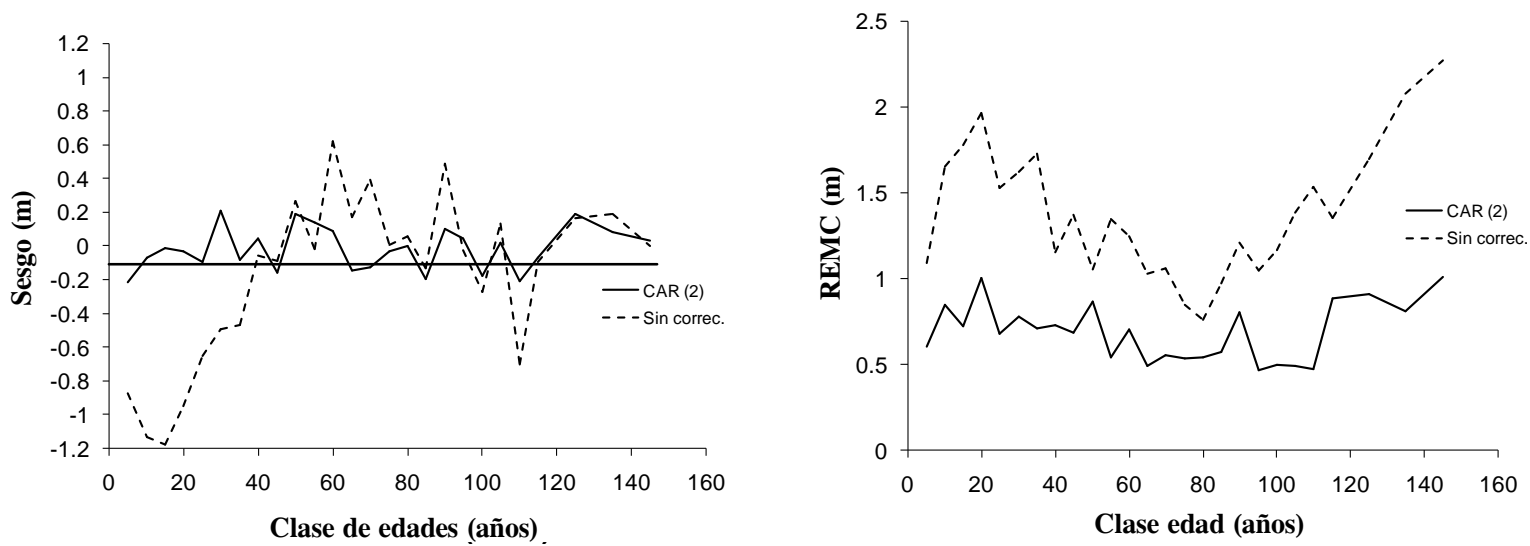

Figura 3. Sesgo y raíz del error medio cuadrático (REMC) en las predicciones de altura estimadas con la Ec. 6 sin corrección (línea punteada) y al considerar la corrección de la autocorrelación con un modelo autorregresivo de segundo orden (CAR(2)) (línea sólida).

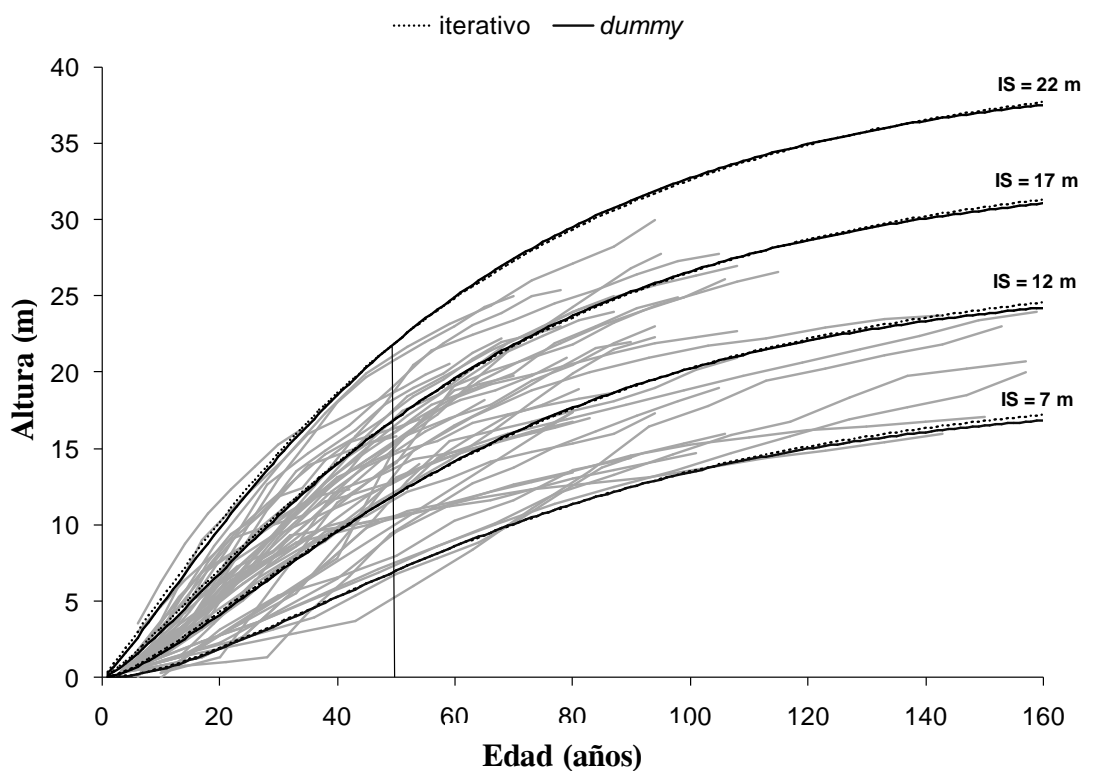

Figura 4. Comparación de las curvas para índices de sitio de $7,12,17$ y $22 \mathrm{~m}$ a la edad de referencia de 50 años (línea vertical), generadas con la Ec. $9\left(H_{1}=H_{0} \cdot\left[\frac{1-e^{-0.0174_{1}}}{1-e^{-0.0174_{0}}}\right]^{-1.905091 .2228 X}\right)$ con el procedimiento de variables dummy (línea sólida) y el procedimiento iterativo (línea punteada) con corrección de la autocorrelación con un modelo autorregresivo de segundo orden (CAR(2)). 
Con ambos procedimientos de ajuste se generan curvas similares, como lo indica la Figura 4 que representa las curvas ajustadas con la corrección de la dependencia de los residuos para diferentes índices de sitio sobrepuestas en las trayectorias de las alturas observadas a través del tiempo y en los estadísticos de bondad de ajuste (Cuadro 2). Sin embargo, aunque ambos métodos son conceptualmente iguales y en condiciones normales alcanzan estimaciones casi idénticas (Cieszewski et al., 2000; Krumland y Eng, 2005), se recomienda utilizar el método iterativo ya que el método dummy es adecuado para bases de datos de tamaño pequeño, p. ej. hasta 200 análisis troncales, mientras que para bases de datos mayores es preferible el método iterativo.

La formulación GADA del modelo base de ChapmanRichards (Ec. 1) al considerar $a_{1}$ y $a_{3}$ como parámetros dependientes de la productividad del sitio e incluir la estructura del error mediante el procedimiento iterativo, es:

$$
H_{1}=H_{0} \cdot\left[\frac{1-e^{-0.0174_{1}}}{1-e^{-0.0174_{0}}}\right]^{-1.905911 .2228 X}
$$

donde $H_{l}$ es la altura predicha (m) a la edad $t$ (años), y

$$
\begin{gathered}
X=\frac{1}{2}\left\{\ln \left(H_{0}\right)+1.9050 L_{0} \pm \sqrt{\left[-1.9050 L_{0}-\ln \left(H_{0}\right)\right]^{2}-4 \cdot 11.2228 L_{0}}\right\} \\
L_{0}=\ln \left[1-e^{\left(-0.0174 t_{0}\right)}\right]
\end{gathered}
$$

donde $H_{0}$ y to representan la altura dominante y la edad iniciales. Nótese que los parámetros locales son descartados de la misma forma que lo son los coeficientes de autocorrelación, como lo propuso Cieszewski (2001), debido a que el uso general del modelo considera la realización de predicciones con base en la altura observada y su edad asociada en nuevos individuos. Del mismo modo, Huang (1999) y Parresol y Vissage (1998) indicaron que en la expresión definitiva de los modelos generalmente se ignoran los valores de los parámetros de la estructura de autocorrelación del error $\left(\rho_{i}\right)$, debido a que el principal propósito de su uso es obtener estimaciones de los parámetros 6 del modelo insesgados y más eficientes; además, en la práctica no se conocen los errores $e_{i-1} \mathrm{o} e_{i-2}$ sin un análisis troncal.

Para usar el modelo GADA ajustado en la estimación de la altura dominante $H$ de una masa a una edad $t$ determinada, dados un índice de sitio $I S$ y su correspondiente edad de referencia $t_{\text {ref }}$ asociada, basta con sustituir $H_{l}$ por $H, t_{l}$ por $t, H_{0}$ por $I S$ y $t_{0}$ por $t_{r e f}$ en la Ec. 9. De manera similar, para estimar el índice de sitio a partir de un par altura dominante-edad dado $(H-t)$, basta con sustituir $H_{l}$ por $I S, t_{1}$ por tref, $H_{0}$ por $H$ y to por $t$ también en la Ec. 9.

\section{CONCLUSIONES}

El empleo de modelos invariantes en edad de referencia proporciona gran flexibilidad, ya que se pueden adaptar a cambios de manejo que requieran la modificación de la edad de referencia. La ecuación aquí obtenida $\left(H_{1}=H_{0} \cdot\left[\frac{1-e^{-0.01744_{1}}}{1-e^{-0.01744_{0}}}\right]^{-1.9050+1.2228 X}\right)$ puede utilizarse para cualquier edad de referencia sin afectar las predicciones de la altura o del índice de sitio para una calidad dada. Con estos modelos, las estimaciones de altura y de índice de sitio se obtienen directamente, sin necesidad de utilizar procedimientos iterativos.

\section{AGRADECIMIENTOS}

Al Consejo Nacional de Ciencia y Tecnología, por el apoyo otorgado a través del Programa de Apoyos Complementarios para la Consolidación Institucional de Grupos de Investigación, Modalidad: Retención (Convenio No. 103645). A las instituciones participantes en este estudio que forman parte del proyecto FONCICYT No. 92739. A los comentarios del editor y de dos revisores anónimos que contribuyeron de manera importante a mejorar la primera versión de este trabajo.

\section{BIBLIOGRAFÍA}

Bailey R L, J L Clutter (1974) Base-age invariant polymorphic site curves. For. Sci. 20:155-159.

Cieszewski C J (2001) Three methods of deriving advanced dynamic site equations demonstrated on inland Douglas-fir site curves. Can. J. For. Res. 31:165-173.

Cieszewski C J (2002) Comparing fixed- and variable-base-age site equations having single versus multiple asymptotes. For. Sci. 48:7-23.

Cieszewski C J (2003) Developing a well-behaved dynamic site equation using a modified Hossfeld IV function $\mathrm{Y} 3=(\mathrm{axm}) /(\mathrm{c}+\mathrm{xm}-1)$, a simplified mixed-model and scant subalpine fir data. For. Sci. 49:539-554.

Cieszewski C J (2004) GADA derivation of dynamic site equations with polymorphism and variable asymptotes from Richards, Weibull, and other exponential functions. Univ. of Georgia PMRC-TR 2004-5. Athens, Georgia. 16 p.

Cieszewski C J, R L Bailey (2000) Generalized Algebraic Difference Approach: Theory based derivation of dynamic site equations with polymorphism and variable asymptotes. For. Sci. 46:116126.

Cieszewski C J, M Harrison, S W Martin (2000) Practical methods for estimating non-biased parameters in self-referencing growth and yield models. Univ. of Georgia PMRC-TR 2000-7. Athens, Georgia. $12 \mathrm{p}$.

Clutter J L, J C Fortson, L V Pienaar, H G Brister, R L Bailey (1983) Timber Management: A Quantitative Approach. John Wiley \& Sons, Inc. New York. 333 p. 
Corral-Rivas J J, J G Álvarez, G A Ruiz, K Gadow (2004) Compatible height and site index models for five pine species in El Salto, Durango (Mexico). For. Ecol. Manage. 201:145-160.

Diéguez-Aranda U, J A Grandas-Arias, J G Álvarez-González, K Gadow (2006) Site quality curves for birch stands in NorthWestern Spain. Silva Fennica 40:631-644.

Fabbio G, M Frattegiani, M C Manetti (1994) Height estimation in stem analysis using second differences. For. Sci. 40:329-340.

Goelz J C G, T E Burk (1992) Development of a well-behaved site index equation: jack pine in north central Ontario. Can. J. For. Res. 22:776-784.

Gregoire T G, O Schabenberger, J P Barrett (1995) Linear modelling of irregularly spaced, unbalanced, longitudinal data from permanent-plot measurements. Can. J. For. Res. 25:137-156.

Huang S (1997) Development of a Subregion-based Compatible Heightsite Index-age Model for Black Spruce in Alberta. Alberta Land and Forest Service, Forest Management Research Note $\mathrm{N}^{0} 5$, Pub. $\mathrm{N}^{\mathrm{o}} \mathrm{T} / 352$. Edmonton, Alberta. $55 \mathrm{p}$.

Huang S (1999) Development of compatible height and site index models for young and mature stands within an ecosystem-based management framework. In: Empirical and Process-based Models for Forest Tree and Stand Growth Simulation. A Amaro, M Tomé (eds). Lisboa. pp:61-98.
Krumland B, H Eng (2005) Site Index Systems for Major YoungGrowth Forest and Woodland Species in Northern California. The Resources Agency. Calif. Dept. of Forestry and Fire Protection. California Forestry Report $\mathrm{N}^{\circ}$ 4. State of California. $220 \mathrm{p}$.

Monserud R A (1984) Height growth and site index curves for inland Douglas-fir based on stem analysis data and forest habitat type. For. Sci. 30:943-965.

Parresol B R, J S Vissage (1998) White Pine Site Index for Southern Forest Survey. Res. Pap. SRS-10. U.S. Department of Agriculture, Forest Service, Southern Research Station. Asheville, NC. 8 p.

Richards F J (1959) A flexible growth function for empirical use. J. Exp. Bot. 10:290-300

SAS Institute Inc (2004) SAS/ETS User's Guide, Version 9.1. Cary, NC, USA. $1315 \mathrm{p}$

Tait D E, C J Cieszewski, I E Bella (1988) The stand dynamics of lodgepole pine. Can. J. For. Res. 18:1255-1260.

Van Laar A, A Akça (2007) Forest Mensuration. Managing Forest Ecosystems, Volume 13. Springer. $383 \mathrm{p}$.

Zimmerman D L, V Núñez-Antón (2001) Parametric modelling of growth curve data: An overview (with discussion). Test 10:173. 